\title{
Security and Surveillance System for Drivers based on User Profile and Learning Systems for Face Recognition
}

\author{
Loubna Cherrat \\ Mathematic and Application Laboratory, FSTT of Tangier \\ Tangier (Morocco) \\ Tel: 06-64-43-39-18Ｅ-mail: cherratloubna2@gmail.com \\ Mostafa Ezziyyani \\ Mathematic and Application Laboratory, FSTT of Tangier \\ Tangier (Morocco) \\ Tel: 06-61-63-03-01 E-mail: ezziyyani@gmail.com \\ Annas EL Mouden \\ Mathematic and Application Laboratory, FSTT of Tangier \\ Tangier (Morocco) \\ Tel: 06-66-63-73-63 E-mail: Elmouden.annas@gmail.com \\ Mohammed Hassar \\ Mathematic and Application Laboratory, FSTT of Tangier \\ Tangier (Morocco) \\ Tel: 06-55-65-76-11Ｅ-mail: m.hassar@gmail.com
}

Received: February 23, 2015

DOI: 10.5296/npa.v7i1.7151
Accepted: March 23, 2015 Published: April 30, 2015

URL: http://dx.doi.org/10.5296/npa.v7i1.7151 


\begin{abstract}
Despite the large and spectacular development in the field of vehicle safety, particularly in the context of driver safety needs, solutions remain insufficient and independent. In this paper, we propose a new system that has been dubbed 3SD "Security and Surveillance System for Drivers". It is a multifunction system as a complete package based on intelligent sensors and cameras that constantly monitor the vehicle's environment and the behavior of the driver to detect early so potentially dangerous situations. In critical driving situations, these systems alert and actively help the driver; if necessary, they automatically intervene to prevent or mitigate the consequences of an accident. The package proposed includes an application comprising a set of pre-registered drivers in a specialized social network interconnected to a geolocation server for distributed real-time sharing of information and data useful for security and traffic. The system is based mainly on learning systems for face recognition based on advanced algorithms Viola and Jones, PCA and management of drivers profiles based on preferences to provide the following features: early detection of sleep, unconsciousness and poor driver behavior, security against theft of vehicles, driver comfort and control and sharing of traffic information in real time between the conductors.
\end{abstract}

Keywords: Security, Surveillance, Detection, Behavior, mobile communication, geolocation, Web Service control systems.

\title{
1. Introduction
}

Facial recognition is part of the broader field of computer vision. The principle of facial recognition is very simple: it is to identify a given face to use and more specifically for safety. Identification can take many forms: it can be to determine who owns a face to determine the owner of an object (eg a car owner), to decide whether or not the face is recognized, or even in some cases to determine the characteristic of a face (the emotion or eye condition). Facial recognition methods employing the technique of principal component analysis, which marks a notable difference from the more classical methods, called geometric or local methods, which are based on the particularities of the analyzed face and whose defects are its lack of precision, and sensitivity to information that is not relevant. The method we used is described as global, since the entire face is then analyzed. Our recognition technique will therefore use the method of analysis in principal components (PCA) for Security and Surveillance System for Drivers [1][2].

The remainder of this paper is organized as follows: in the first part, we present the related work, and Viola/Jones algorithm used to develop our system 3SD. Then we present general context of "Security and Surveillance System for Drivers" project . Then, the second part presents the analysis and design of the project, beginning with defining the main features. Then, in the third part, we present the platform with an explanation of the different components that we have developed. Finally, we describe the system and performed services.

The remainder of this paper is organized as follows. In Section 2 we review the concept and principle of Face Recognition Technology. Section 3 discusses the Viola and Jones 
algorithm that fits to our application for face recognized. The Study the effectiveness of the system and show experimental results were provided in Section 4. The specification and Background of the Project of our system is provided in Section 5 and 6 . Then the sections 7 we draw the Functional architecture of the system. Finally we conclude with Section 8

\section{Face Recognition Technology}

Automatic face recognition involves three main steps: (1) Face detection, (2) extraction and normalization of facial features, (3) identification and / or verification. Some image processing techniques may be common to several steps [3][4]. For example, the extraction of facial features (eyes, nose, mouth) is used both for detection and for the identification of faces. Furthermore, the face detection steps and feature extraction can be executed concurrently. It depends particularly on the nature of the application, the size of the training set, and the conditions of shooting [3][4]. In our case the size of the training set is medium in size with the shooting condition is controlled. Subsequently we present the two most recommended approaches adapted to our application domain for road safety:

\subsection{Approaches based on knowledge}

This methodology is interested parties facial features such as the nose, mouth and eyes. It is based on the definition of strict rules from the relationship between facial features. These methods are primarily designed for face localization. In [5][6], the authors use a rule-based method. First, the facial features are located using the projection method to detect edges of a face. To do this, starting with the low-resolution images, a set of face candidates is determined by a set of rules to check for uniform regions in an image. The face candidates are then verified by searching the existence of prominent facial features by calculating local minima at higher resolutions [7]. An interesting feature of this technique "top down" interest search area is to reduce the computation time required by the use of images downsampled. Unfortunately, this technique causes many false detections and a low rate of detection.

\subsection{Approaches based on the "Template-matching"}

The templates can be defined to be "manually" or using parameterized functions. The idea is to calculate the correlation between the candidate image and the template. These methods still encounter some robustness issues related to changes in light. This technique uses a set of invariants describing the model's face. To determine the invariants to changing light conditions to characterize the different parts of the face (such as eyes, cheeks, and forehead); this algorithm and calculates the luminance ratios between the areas of the face and retains the directions of these reports.

Learning is performed on a very wide range of positive images (that is to say containing the object) and negative (not containing the object). Thousands of examples are usually required. This training includes:

- The calculation of pseudo-Haar features [7] on the positive and negative examples. 


\section{Macrothink}

- The drive of the cascade to each stage of the cascade, a strong classifier is trained by AdaBoost. It is built up by successively adding weak classifiers trained on one characteristic, to obtain consistent performance to the detection rate and false alarm for the desired floor.

Figure 1 shows the process of the algorithm used for data stored in an XML file and how to classify each image location [9].

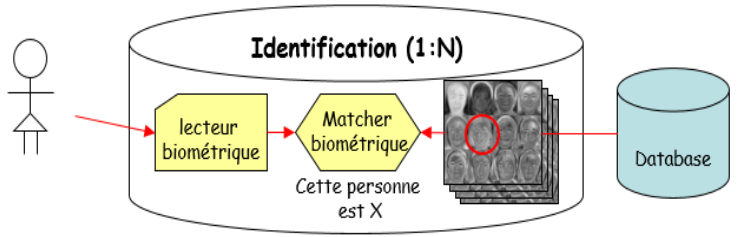

Figure 1. How PCA works

\subsection{Principal Process of Face Recognition}

The principal component analysis (PCA) [8] was invented in 1901 by Karl Pearson. Now, it is mainly used as a tool in the analysis of data and for the production of predictive models (Figure 2). PCA is a mathematical procedure which uses orthogonal transformation to convert a set of observations of correlated variables to a set of values of linearly uncorrelated variables called principal components. The number of principal components is less than or equal to the number of original variables. This transformation is defined such that the first principal component is the largest possible variance and each succeeding component in turn the highest possible variance in the constraint it is orthogonal (ie, without correlation with the previous components). The main components are guaranteed to be independent only if the data set is normally distributed jointly. PCA is sensitive to the relative scaling of the initial variables [9].

Generally, the main steps to follow are:

- Face detection.

- Extraction characteristics.

- Classification of facial images.

The characteristics considered are:

- Local features: eyes, nose, and mouth.

- Global Properties: feature extraction of the entire image. 


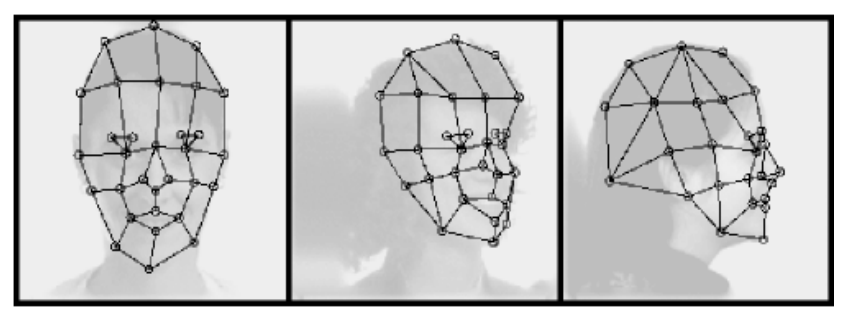

Figure 2. Global Features

The "Eigenfaces" and "Fisherfaces" are two basic methods in the proposed approaches for face recognition. Both are based on the decomposition of the image on a reduced space and in the search for an optimal feature vector describing the image of the face to be recognized. The facial images are linearized as vectors $\mathrm{x}$ and are expressed as a linear combination of orthogonal basis reduced to a selected dimension m. Principal Component Analysis PCA or the first face recognition system has yielded results using the method of "Eigenfaces " [10].

\subsection{Classification of facial images}

\subsubsection{Feature extraction}

PCA is a mathematical procedure that uses an orthogonal transformation to convert a set of possible values of a correlated variable $\mathrm{M}$ to a set of values of an uncorrelated variable $\mathrm{K}$ called principal components (Figure 3)[11][12].

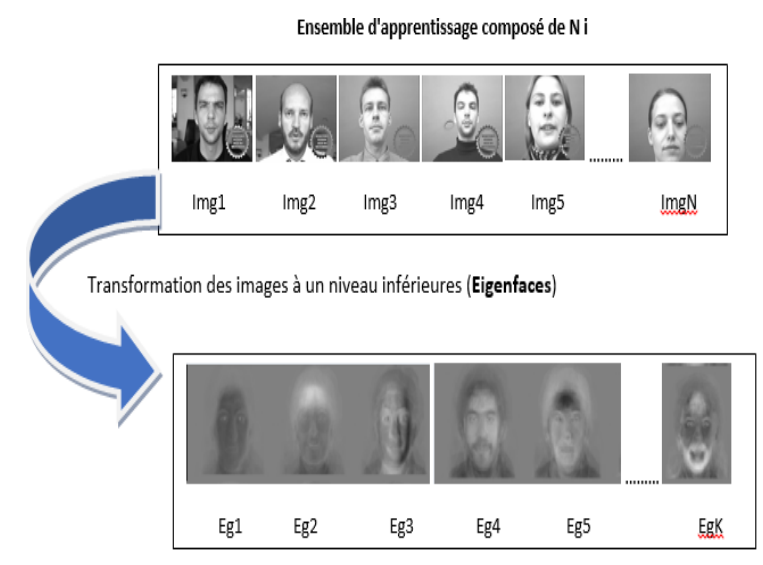

Figure 3. Image Classification

\subsubsection{Classification by characteristics}

This transformation is defined such that the first principal component has the most dominant characteristics of the data set and each succeeding component in turn has the following characteristics most dominant possible, under the constrained that it be not correlated with the previous components. The following Figure 4 show the efficacy of the clarification by characteristics: 


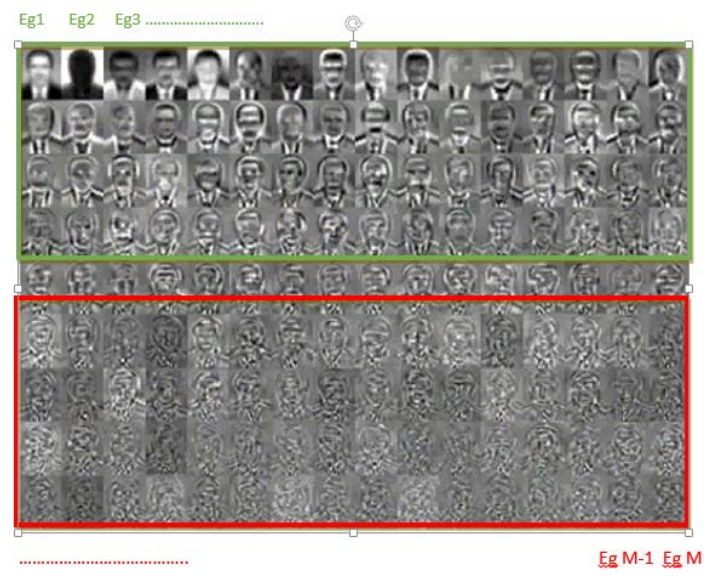

Figure 4. Classification by characteristics

\subsection{Viola and Jones algorithm}

Viola and Jones [13] is a method of object detection in a digital image, proposed by researchers Paul Viola and Michael Jones in 2001. It is one of the first methods that can effectively detect objects in an image on real-time [14]. Originally invented to detect faces, it may also be used to detect other types of objects such as cars or aircraft. The Viola and Jones method is one of the best known and most widely used methods, especially for face detection and people detection.

\subsubsection{Method Principle}

Viola and Jones method is an approach based on appearance which consists in browse the entire image by calculating a number of characteristics in rectangular areas that overlap. It has the peculiarity to use very simple but very many features. One innovation of the method is the introduction of integral images that allow rapid calculation of these characteristics. A second important innovation is the selection of these characteristics by boosting and interpreting the features as classifiers. Finally, the method proposes an architecture for combining the boosted classifiers in a cascade process, which provides a net gain in detection time. It is divided into two steps: a step of learning of based on a large number of positive examples (that is to say, the objects of interest, such as faces) and negative examples, and detection phase by applying this classifier to unknown images.

\subsubsection{Characteristics}

Viola and Jones proposed to use the characteristics, that is to say, a concise and informative representation, calculated from the values of the pixels. Viola and Jones define very simple features, the Haar-like features, which are calculated by the difference of the sums of two pixels or more adjacent rectangular regions. The figure below shows examples of characteristics proposed by Viola and Jones to 2, 3 or 4 rectangles, in which the amount of dark pixels is subtracted from the sum of the white pixels (Figure 5). 


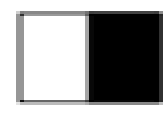

(1)

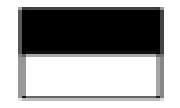

(2)

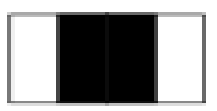

(3)

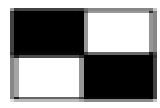

(4)

Figure 5. HaarTraining Rectangles

\subsubsection{Selection of characteristic by boosting method}

The second key element in the method of Viola and Jones is the use of a boosting method to select the best characteristics. Boosting is a principle which is to build a "strong" classifier from a weighted combination of 'weak' classifiers, that is to say, giving on average a better answer than a random draw. Viola and Jones adapt this principle by equating a feature to a weak classifier, building a weak classifier which uses only one characteristic. Learning the weak classifier is then to find the threshold value of the characteristic to better separate the positive examples of negative examples (Figure 6). The classifier is then reduced to a couple (characteristic threshold).

This scheme is an illustration of the architecture of the cascade: windows are sequentially processed by the classifiers, and discarded immediately if the answer is negative (F).

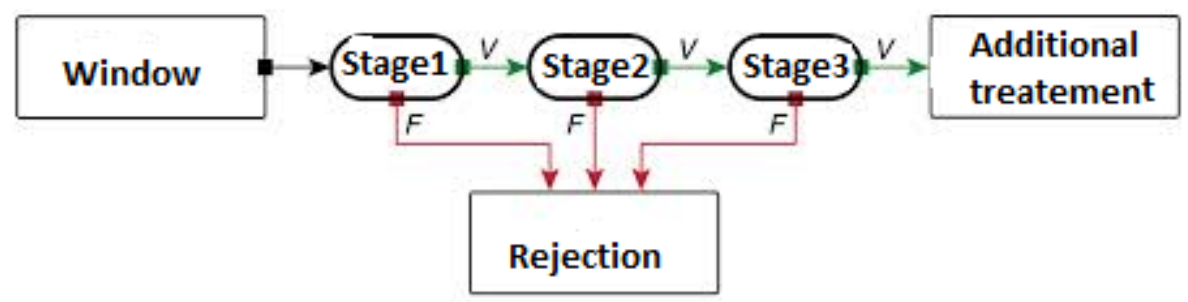

Figure 6. Boosting method

\section{Study the effectiveness of the system}

Recognition of our solution is the most robust and optimal solution. At the forefront of technology, our facial recognition algorithms are optimal for work on images of faces, especially image processing, pattern recognition and computer vision. The accuracy and reliability of the recognition tool determine the quality of facial recognition systems. Through tests carried out, we have demonstrated that our algorithm exceeds the performance of the algorithms independent suppliers. In addition, they showed a higher independence to facial differences such as pose, mimic, the variance of age, different hair styles, glasses or temporary lighting changes.

\subsection{Experimental results}

The experiment shows that all of the methods were capable of determining faces, and the Viola-Jones method is able to find image, with separate training and with a high level of precision. The results of the experiment for the implemented algorithm, including the average time to complete a single image in the database and the accuracy against the tested database. 
A comparison of the time should be taken cautiously. The accuracy of the Viola-Jones algorithm remains fairly consistent between each of the databases, approaching $90 \%$. The accuracy of other algorithms is no better than those shown in the Viola-Jones. Though this is certainly a means of finding a face in an image.

Though the algorithm is fast, it is incapable of finding faces in the visible spectrum. This is due to the intensity of the background and face colors being the same. The algorithm was designed for detection of car driver for security, where the face would be significantly brighter than the background.

Table 1. Classifiers average of run time (s) and accuracy (in \%) in terms of true detection (TD) and false detection (FD) rate.

\begin{tabular}{|c|c|c|c|c|}
\hline \multirow[b]{2}{*}{ Objects } & \multicolumn{4}{|c|}{ Database size } \\
\hline & $\begin{array}{c}\text { Less than } 500 M B \\
\text { TD-FD-RT }\end{array}$ & Between & $\begin{array}{l}500 \mathrm{MB} \text { and } 10.000 \mathrm{MB} \\
\text { TD-FD-RT }\end{array}$ & $\begin{array}{c}\text { More than } 10000 \text { MB } \\
\text { TD-FD-RT }\end{array}$ \\
\hline Face & 89-0.05- 1.7 & & 97-0.00-1.99 & $99.5-0.00-2.20$ \\
\hline Eye & 86-0.04-1.3 & & $98-0.00-2.09$ & $98.9-0.00-2.10$ \\
\hline
\end{tabular}

Table 1 shows final results of Face have a high accuracy related to eye detection for all size of database both for detection (TD) and false detection (FD). And the accuracy increases in proportion to the size of the database. So, the evolution of the performance of the detector according to the size of the database is taken into account. Although the differences are limited, we can make two observations. On the one hand, use a very small data base does not provide the best results, confirming that the databases which cover a large part of the image are relevant for detecting the face and the eyes. On the other hand, the worst results are obtained by minimizing the database. We must find a compromise on the optimal size.

Another indication in Table 1 shows the execution time required for the face detection phase and eyes detection stored in a database, and quantification of the image description, using whole database. The performance of the detector according the size of the database used is interesting if the average time based detection is prohibitive for any large-scale use. Accelerated version offers reasonable execution time, in addition to greater accuracy if we neglect the necessary learning phase out pre-processing, extraction and quantification.

\section{Background of the Project}

Fatigue, sleep and poor driving behavior can be described as a progressive decline in mental and physical alertness that leads to drowsiness and sleep. The degree of fatigue is problematic from the moment it compromises the ability of a driver to perform tasks that require attention and judgment, and that appeal to his reflexes. In general, the main known causes of fatigue at the wheel are:

- $\quad$ Linked to the driver: The circadian rhythms (biological clock), the state of health 
(physical and mental), the quantity and quality of sleep, wake time, food, fitness, family life and age, etc.

- Related work: corporate culture, time of day, length of the period of work, lack of adequate rest periods, rotating shifts, night work and the importance of mental workload or physics, etc.

- Related to the environment: The ergonomics of the vehicle, the type of travel, road and weather conditions, availability of rest areas, the monotony of the road and environmental stress (heat, noise and vibration), etc.

The consequences of fatigue greatly impair driving performance. Indeed, there is a strong connection between fatigue and errors that lead to an accident: Fatigue increases the reaction time, decreases awareness, impairs judgment, impairs memory, reduces the field of view and increases risk of drowsiness and sleep. To solve this problem, the proposed solutions are generally limited to the analysis of the particular facts of certain behaviors, for example, if the driver no longer turns the steering wheel for a short time and then makes a sharp correction trajectory. In this paper, we propose the generalization of the solutions on all possible pre behaviors that can often be a sign of deconcentration and increased fatigue based on facial expressions or movements. The warning device for the driver that we propose is an intelligent system that monitors all the bad driving behavior. In case of abnormal conduct or detection of fatigue expressions on the face of the driver, it will be alerted by a signal and a message on the screen to recommend to pause or by performing certain actions in order to attract the driver's intention namely; automatic opening of windows, increasing the volume or change the radio channel, change the layout or the vibration of the seat or the mandatory stop in the next rest area by the automatic pump shutdown injection fueled by calculating the required depending on the remaining distance etc.

Another problem that we will discuss for an integrated solution is the protection of vehicles against theft. Car theft is a global problem and very expensive. Indeed, several solutions are available but are still very limited and insufficient against theft crime that continues to increase. In this perspective, we propose an innovative solution based on the detection of persons authorized to drive the vehicle.

The third category of problems related to the safety of drivers arises in the context of safety in the road. It is the sharing of information between the conductors. Information sharing between drivers via a dedicated social network is an effective way to road safety and to reduce the accident rate.

Thereafter, we will propose a detailed study of an adequate and effective solution to these three problematic in a single package consisting of:

- An embedded system in the vehicle with three cameras and special sensors for security against theft, self-regulation of the vehicle devices to the profile of the driver and for the detection and control of sleep and bad behavior.

- An application to oversee the security process through a web service. 


\section{Macrothink}

- A social network for the dissemination and sharing of information between the drivers in real time.

- A Mobile application, used by the police officers that offers the ability to view alerts sleep or theft of vehicles in order to arrest the suspects.

\section{Functional study of the 3SD physical components}

The system services that we offer are mainly based on the direct control of the driver. Therefore, the automatic identification of the driver and those exact behaviors and knowledge of the state of the external environment via high-resolution cameras are the basic key for our solution. To achieve this objective, the device, which we propose, has three cameras: the first focused on the driver to record and filter images on the driver's face in a local database. The second camera allows controlling the channel outputs through viewing the road during the traffic in order to maintain the path of the vehicle according to the markings and warn the driver in case of involuntary crossing. The third camera is used to record the state of the external environment for possible communication and sharing with a network of drivers all the information on road conditions, climate etc....

To meet safety requirements, the 3SD system analyzes these images using the Viola and Jones algorithm considered as one of the most important methods for object detection and as a method of supervised learning. Secondly we will use the principal component analysis (PCA). In addition we use emerging technologies in mobile communication, geolocation, Web Service and control systems. Another component in the integrated 3SD device allows detecting the accident via the automatic calculates the degree of shock and degradation of speed. This component (Accelerometer) which is associated with a location-based map is used to communicate the geographical position of the accident in real time.

\section{Analysis and specification}

\subsection{Main functionalities}

This section is reserved the enumeration and the description of the different features that the system must ensure 3SD. After a thorough analysis, we came to identify the following features:

- Management of drivers, vehicles and accounts: Every vehicle owner can perform different managements to add, modify and delete vehicle users and accounts.

- Security against theft: Once the camera detects the face of a person who is not registered in the database, a message must be sent to informing the phone owner that a person is using his car so that it notifies the agency in order to locate the vehicle and report it to the police. Figure 7 present the process of security for car protection and against theft (1:detection, 2-3:identification,4-10:notification,5-6-7:traitment, 8-9: localization) 


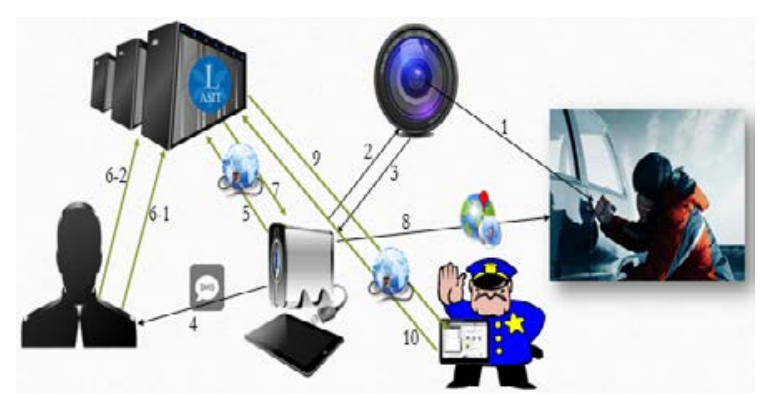

Figure 7. Security process against theft

- Sleep detection and driver behavior: To enable detection of the eyes, the vehicle speed must be greater than or equal to $80 \mathrm{~km} / \mathrm{h}$, in this case the camera starts to detect and classify the eyes of drivers (open or closed). If the camera detects that the driver's eyes remain closed more than two seconds, here it begins the process of driver safety with a series of reactions (alarm, radio, electric windows, SMS, Geo location). The process presented at the Figure 8 (1-2:activation, 3:detection, 4:identification, 5-6-7:traitment, 8-9-10: localization, 11-12:notification)

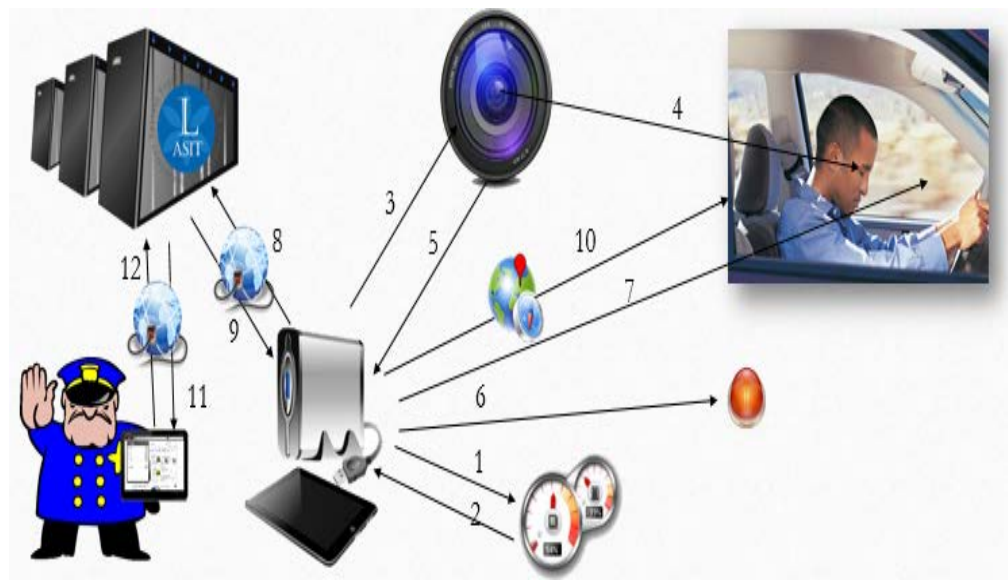

Figure 8. Sleep detection process

- Triggering alarm, radio and electric window: As mentioned earlier, if the camera detects that the driver's eyes remain closed more than 2 seconds, an alarm is initiated, the radio starts, and a power-window opens to wake the driver.

- Sending a text message to a sender: If the camera detects that the driver is in the process of sleep a 2nd time, an SMS is automatically sent to a member of his family (the phone number is preset) to inform that the driver is in danger.

- Sending alert to the server: If the camera detects that the driver is in the process of sleep a 3rd time, an alert should be sent to the remote server (Web Service) to enable the agency to recover the alert in order to locate and report the vehicle.

- Geolocation vehicle: After sending the alert, the web application locates the vehicle with a GPS and seeks the closest station to stop the vehicle or informs the nearest police roadblock to stop it. 
- Social Networks: With our system, the driver can receive offers, notifications, additional services, as himself can share photos, events, alerts (in case of accidents), etc ... All this in a social network that brings together all subscribers of this service.

- Geolocation alerts: Vehicle locating and police roadblocks, continuing alerts and the ability to determine the shortest path of a vehicle at a police checkpoint.

- Data management: The management of subscribers (Add, Delete, Edit, View), font dam management, sales administration and management of alerts.

- Black List: This is a blacklist to record drivers who exceed five alarms, and are considered a danger to others on the road.

\section{Functional architecture of the system}

The functional architecture of the system, as shown in the Figure 9 below, consists of:

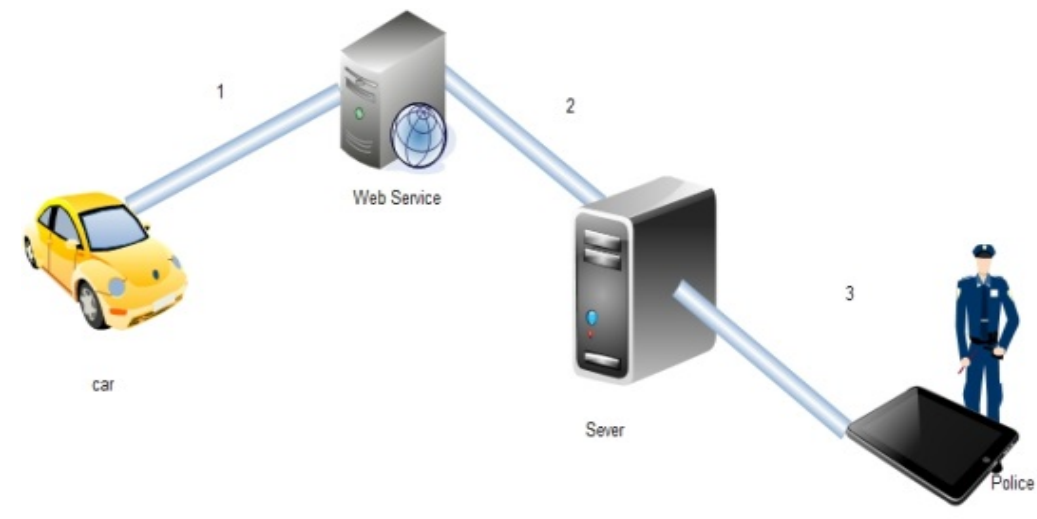

Figure 9. Application architecture

\subsection{Server (web application)}

It is a part used to receive alerts (security or sleeping) with all information regarding the alert type, geographic coordinates, owner information, in order to view this alert in a map to track in real time of this alert.

This section also allows the management of offers before sending them to the driver, as well as the management of subscribers and vehicles.

\subsection{Desktop (Vehicle)}

This part is used to locally treat the security process against theft and sleep of the driver to send an alert regardless of the type of the alert to the server to process it.

\subsection{Web Service}

This part acts as the intermediary between the application of the system and the exchange 


\section{Macrothink}

Network Protocols and Algorithms

ISSN 1943-3581

2015, Vol. 7, No. 1

of information (the case of social network) and sending to server vehicle alerts and sending server alerts to police officers.

\subsection{Mobile Application (Police Officer)}

This part is used by police officers to receive alerts sent by the server and arrest suspects who pose a danger to the road.

\subsection{Social Networks (Social Network)}

In the context of new technologies that have emerged in recent years in the field of automobile manufacturing, we aim to create a complete system that changes the traditional view that says a vehicle is only used to move from a point A to point B. With our system, the driver can receive offers, notifications and additional services, share itself photos, event alerts (in case of accidents), etc. All this is in a social network that brings together all subscribers of this service.

\subsection{Web application (the agency)}

Management of alerts: provides various monitoring operations and traffic alerts dam or the nearest police station, using algorithms known in the domain. For the location of alerts, we use the API V3 Google Maps (Figure 10).

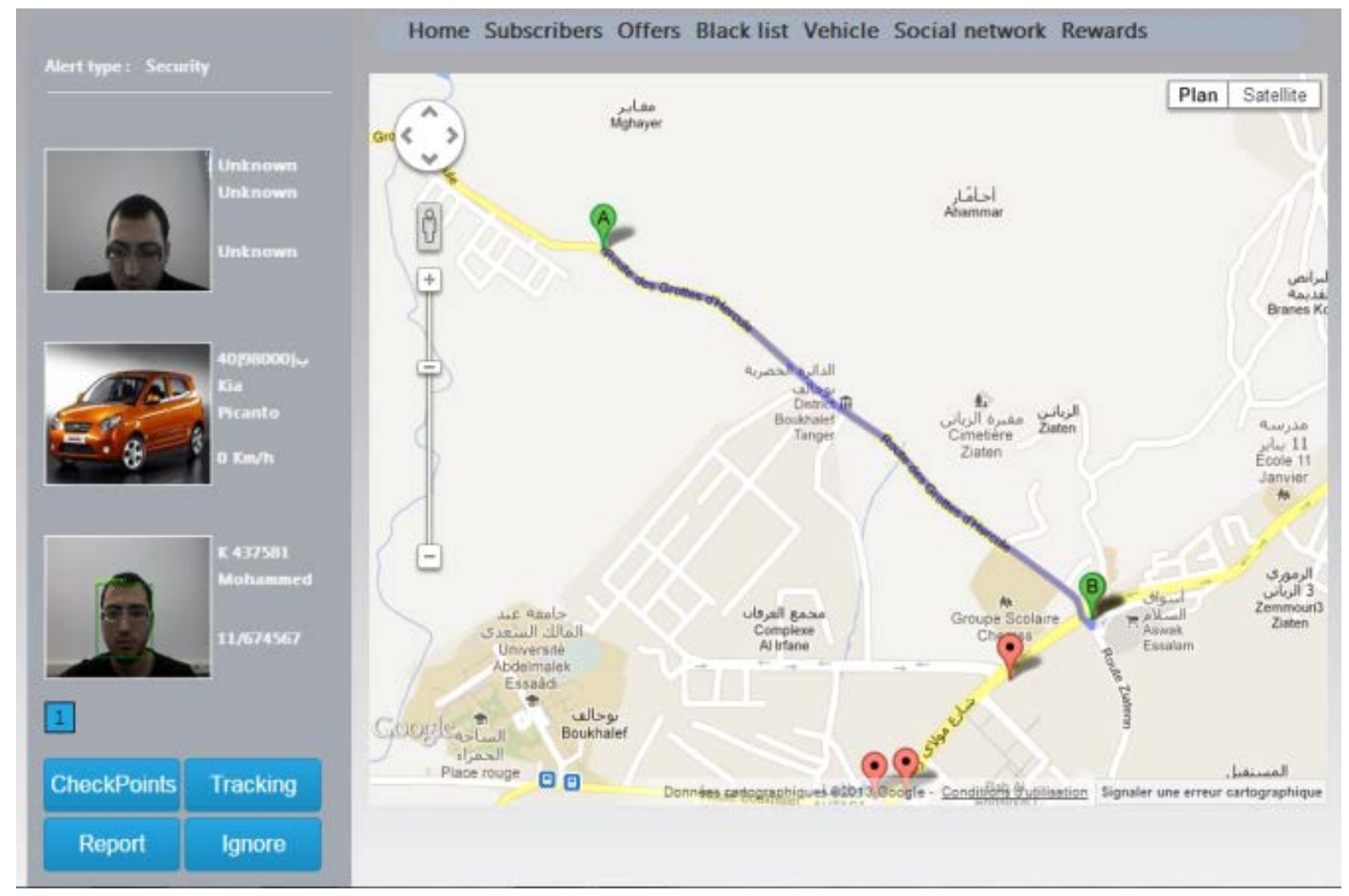

Figure 10. Alert signalling process

- Management of subscribers: This is to ensure that the various managements CRUD (add, edit, delete, view) of subscribers for better control the flow of data alerts, offers and the 
social network.

- Management of vehicles: This task is to ensure the different managements CRUD (add, edit, delete, view) of vehicles to better control the flow of data and alerts subscribers.

- Management of tenders: This part covers the different management's offers that are to notify the subscriber of the latest services. These services include weather, road conditions and many other services.

- Black List: In order to properly control the alerts, we added the principle of Black List. Once an alert is repeated five times, the vehicle is automatically considered as a danger on the road and is recorded in the black list.

\section{7 Mobile Application (Police Officer)}

- Management of alerts: It consists in receiving alerts with all the information on the vehicle, the driver and the real-time vehicle location. All this is in order to stop drivers that can cause traffic accidents.

- Locations of dams / stations: To ensure the real-time location of the police officers, a feature was added to our mobile application that detects the change of coordinates (latitude and longitude) and sends them to the server to update the level of agency.

\subsection{Web Service}

The creation of a web service to ensure communication and data exchange between heterogeneous systems and applications in distributed environments. This is therefore a set of functionality exposed on the internet, by and for the application (the agency) or machines (Vehicles) without human intervention, and synchronously.

\section{Class diagrams and Interfaces}

Class diagrams of Figure 11 and Figure 12 play a central role in the project analysis and design of object-oriented application. They have a set of static model elements, content (internal structure) and their relationship to other elements.

\subsection{Vehicle}

The following diagram shows the class diagram of our desktop application to manipulate the data. 


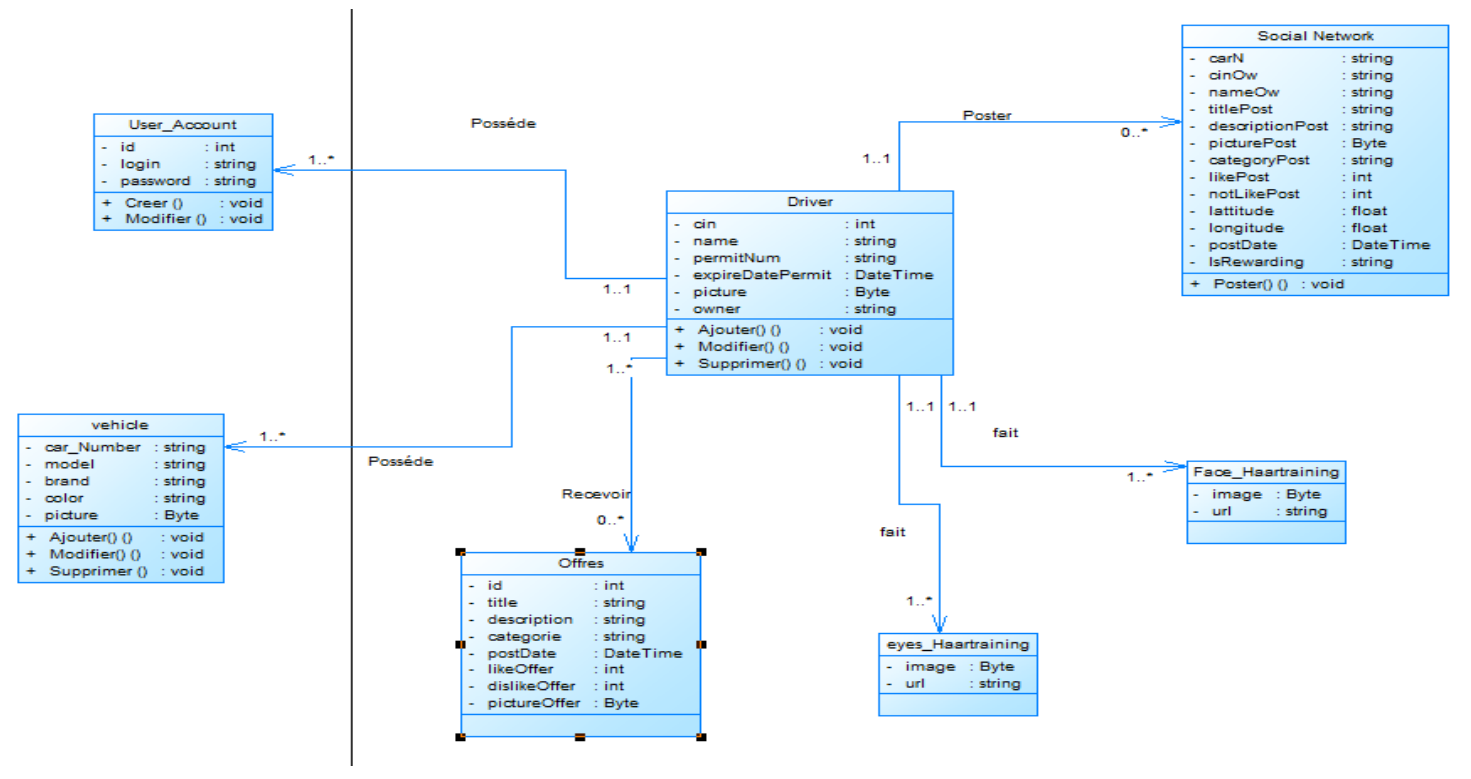

Figure 11. Class diagram for the management of vehicle

\subsection{Agency}

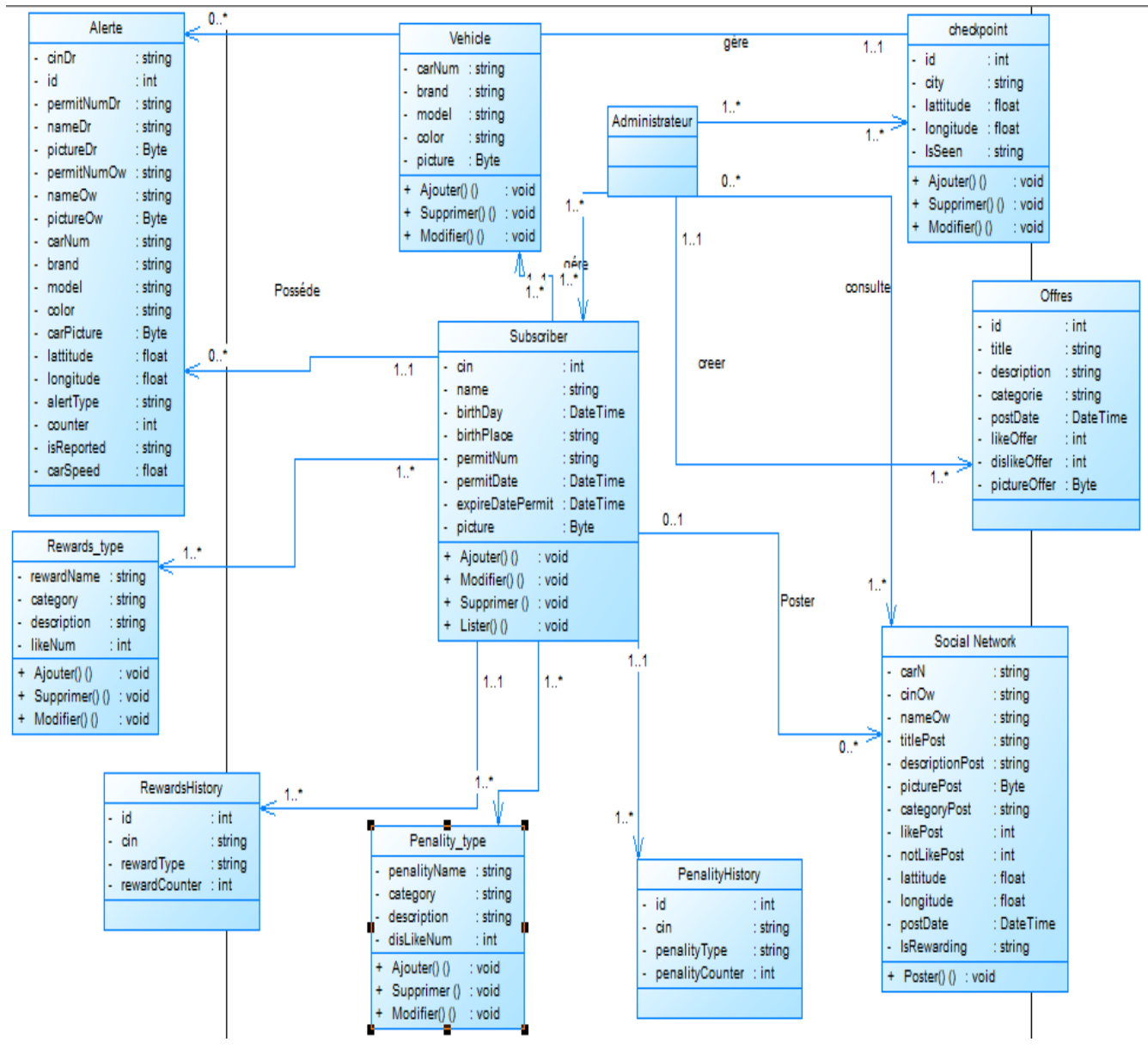

Figure 12. Class diagram for the management of agency 


\section{Macrothink}

Network Protocols and Algorithms

ISSN 1943-3581 2015, Vol. 7, No. 1

Figure 12 shows schematically the interface used for adding a new vehicle with all the necessary information. It also offers the ability to edit or delete the vehicle.

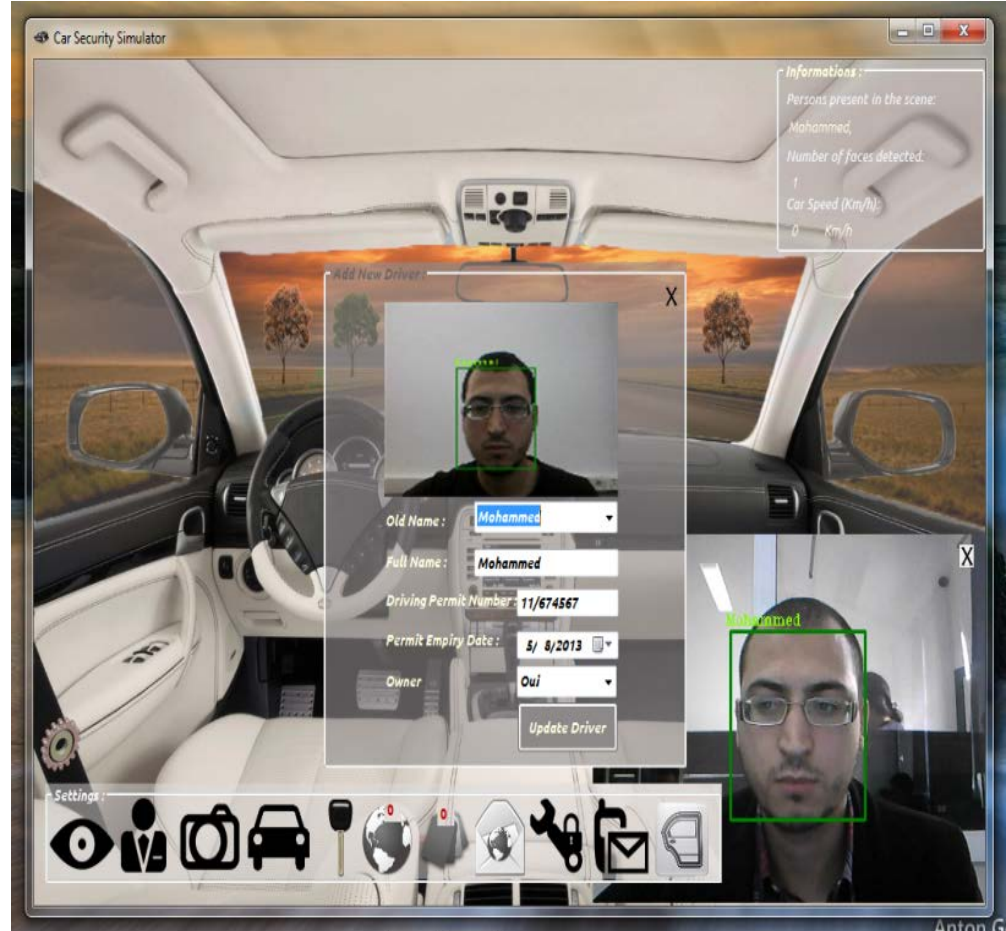

Figure 13. Interface for Vehicle Management

This interface (see Figure 14-a) is used for configuring the GSM modem and the phone number to which to send the SMS alerts.

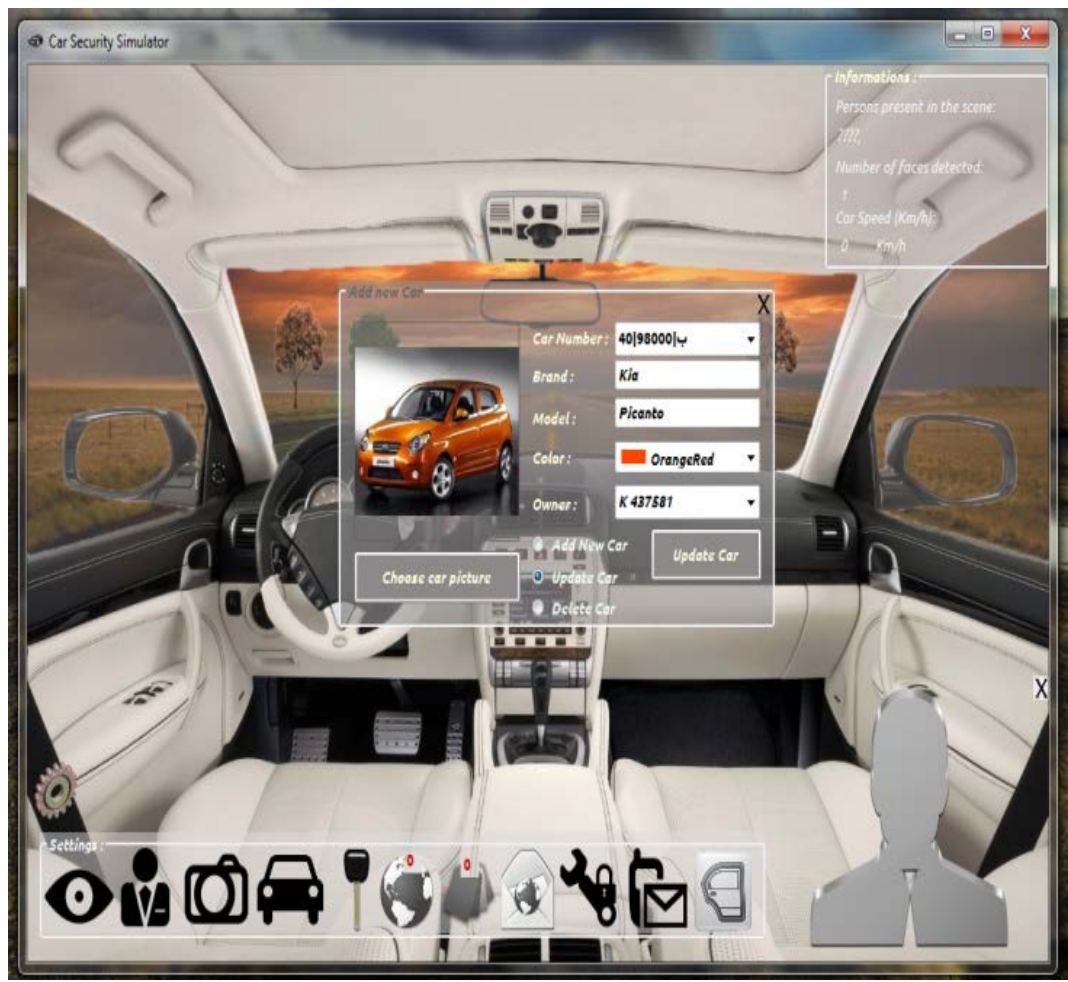

Figure 14-a. Interface configuration 


\section{Macrothink}

This interface (see Figure 14-b) deals with the process of sleep detection. For example, we can see from the figure above that the system detects and classifies the driver's eyes (Open / Closed) after reaching a speed of $80 \mathrm{~km} / \mathrm{h}$.
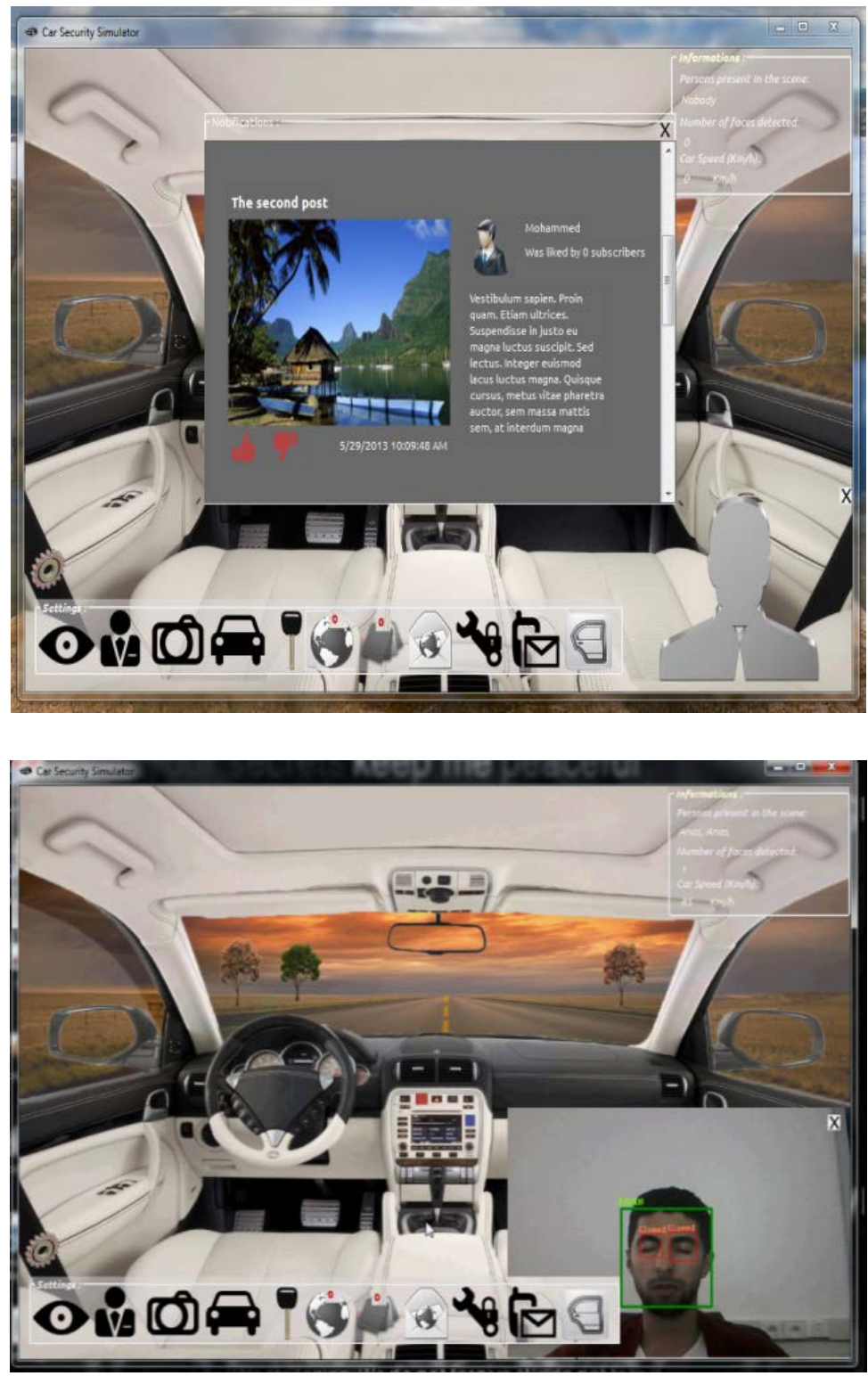

Figure 14-b. Interface for the treatment of the process of sleep detection

Our web application provides the ability to view alerts (sleeping, Security) and geolocation in the map to determine the shortest route alerts to police roadblocks (Figure 14-c).

This application also offers the possibility of managing subscribers, vehicles and fonts dams and many other services which here the host interface: 


\section{Macrothink}

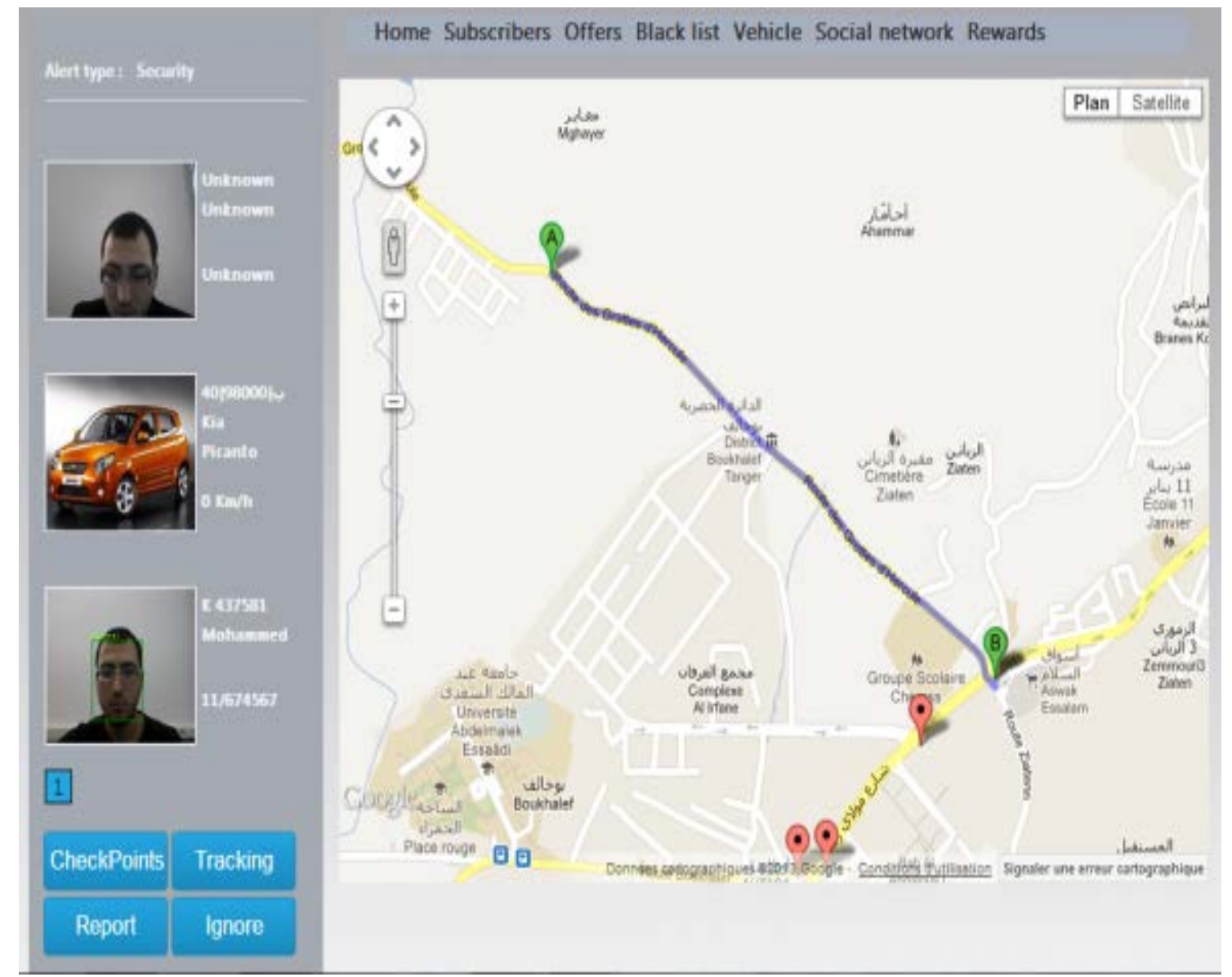

Figure 14-c. Main Interface of 3SD Web application

\section{Conclusion}

In this paper we presented an effective method of face and eyes detection, to link the images stored in the database and faces describing how to drive by the driver. This detection method is both fast and inexpensive in terms of learning, and poses no scale-up problems. To provide these features, the system is based mainly on learning systems for face recognition based on advanced algorithms Viola and Jones and PCA and management of driver profiles based on preference.

As application we have presented in this article 3SD a new system that provides security and monitoring of drivers based on intelligent sensors and cameras that constantly monitor the vehicle's environment and driver behavior to detect a prematurely potentially dangerous situations and automatically intervene to prevent or mitigate the consequences of an accident. Our solution has proposed a package consisting of:

- An embedded system in the vehicle with three cameras and special sensors for security against theft, self-regulation of the vehicle devices to the profile of the driver and for the detection and control of sleep and bad behavior.

- An application to oversee the security process through a web service. 
- A social network for the dissemination and sharing of information between the drivers in real time.

- A Mobile application, used by the police officers that offer the ability to view alerts sleep or theft of vehicles.

As a future work, we plan to address the ambiguities between behaviors, face and eyes . We could group the instances of a single class on visual criteria based on dataming and classification to refine and spread the detection by the forecast notion. The analysis of the face and eyes can also be refined, using the related behavior recognition with the manner of driving: many entities used here are emotions, but we are counting their occurrences separately and those of their definition. We also plan to address the ambiguities between behaviors and emotions named the same frequency, instead of ignoring a basic classification context. Finally, our system could use indexing, image, eyes and behavior to accelerate research and minimize the processing time.

\section{References}

[1] Wang H., Zhou L. B and Ying Y., "A novel approach for real time eye state detection in fatigue awareness system”, In IEEE Robotics Automation Mechatronics (RAM 2010), 28-30, June 2010, Singapore. pp 528-532. http://dx.doi.org/10.1109/RAMECH.2010.5513139

[2] Mahdi Rezaei, Reinhard Klette ,’3D Cascade of Classifiers for Open and Closed Eye Detection in Driver Distraction Monitoring”. Computer Analysis of Images and Patterns, 2011, 14th International Conference, CAIP 2011, Seville, Spain, August 29-31, Volume 6855, pp 171-179. http://dx.doi.org/10.1007/978-3-642-23678-5_19

[3] Langner O., Dotsch R., Bijlstra G. and Wigboldus D. H. J., "Presentation and validation of the Radbound faces”, Database Cognition Emotion, Amsterdam, Pays-Bas, vol. 24, pp. 1377-1388 , 2010. http://dx.doi.org/10.1080/02699930903485076

[4] P. Belhumeur, J. Hespanha, D. Kriegman, Eigenfaces vs. Fisherfaces: Recognition Using Class Specific Linear Projection, IEEE Transactions on Pattern Analysis and Machine Intelligence,New Haven, CT, USA ,vol 19, pp. 711-720, July 1997. http://dx.doi.org/10.1109/34.598228

[5] Kasinski A. and Schmidt A., "The architecture and performance of the face and eyes detection system based on the Haar cascade classifiers”.J. Pattern Analysis Applications, Springer-Verlag, May 2010, vol 13, Poznan, Poland, pp.197-211, 2010. http://dx.doi.org/10.1007/s10044-009-0150-5

[6] Lee K.C. and Kriegman D., “Acquiring linear subspaces for face recognition under variable lighting”, IEEE Trans, Pattern Analysis Machine Intelligence, 21 mars 2005, IL, USA, vol 27,pp.684-698, 2005. http://dx.doi.org/10.1109/TPAMI.2005.92 
[7] K. Messer, J. Matas, J. Kittler, J. Luettin, G. Maître, "XM2VTSBD: The Extended M2VTS database”, AVBPA, Springer Verlag, New York, pp. 72-77, 1999. http://dx.doi.org/10.1177/0956797613503175

[8] Viola P. and Jones M., " Rapid object detection using a boosted cascade of simple features”, IEEE Computer Society Conference on Computer Vision and Pattern Recognition (CVPR 2001), 8-14 December 2001, Kauai, HI, USA. pp. 511-518, http://dx.doi.org/10.1109/CVPR.2001.990517

[9] E. Hjelmas et B. K. Low. "Face detection : A survey",Computer Vision and Image Understanding, 23 October 2000 vol. 83, no. 3, pp. 236-274, 2001. http://dx.doi.org/10.1006/cviu.2001.0921

[10]Lienhart R., Kuranov A. and Pisarevsky V., "Empirical analysis of detection cascades of boosted classifers for rapid object detection”. In Pattern Recognition,Lecture Notes in Computer Science (LNCS) vol 2781, santa Clara CA, USA, pp 297-304, 2003. http://dx.doi.org/10.1007/978-3-540-45243-0_39.

[11] Niu Z., Shan S., Yan S., Chen X. and Gao W., "2D cascaded AdaBoost for eye localization”IEEE,18th International Conference on Pattern Recognition, 2006. In ICPR, vol 2, Hong Kong, pp 1216-1219, 2006. http://dx.doi.org/10.1109/ICPR.2006.1194

[12] Phillips P., Wechsler J., Huang H. and Rauss J., "The FERET database and evaluation procedure for face recognition algorithms”, Image Vision Computing,28 April 1997, Adelphi, MD 20783, USA. Vol 16, 1998. http://dx.doi.org/10.1016/S0262-8856(97)00070-X

[13] Wilson P. I. and Fernandez J., "Facial feature detection using Haar classifiers", Computing Science, April 2006,Consortium for Computing Sciences in Colleges, USA, vol 21, pp.127-133 , 2006.

[14] S. Z. Li, A. K. Jain, "Handbook of Face Recognition", Springer, Springer-Verlag London Limited, London 2004. http://dx.doi.org/10.1007/978-0-85729-932-1

[15] M. Turk, A. Pentland, Eigenfaces for recognition. J. of Cognitive Neuroscience 3, 72-86, 1991. [7]. J. Haddadnia, M. Ahmadi, and K. Faez, "An Efficient Feature Extraction Method with Peudo Zernike Moment in RBF Neural Network Based Human Face Recognition System”, EURASIP JASP, New York, NY, United States, vol. 9, pp. 890-891, 2003. http://dx.doi.org/10.1155/S1110865703305128

[16]Freund Y. and. Schapire R.E, "Experiments with a new boosting algorithm”, In Machine Learning, Florham Park, USA, pp148-156, 1996. 7 May 2002 
http://dx.doi.org/10.1006/jcss.1997.1504

[17]Jesorsky O., Kirchberg K. and Frischholz R., "Robust face detection using the Hausdor distance”, Third International Conference on Audio Video-based Person Authentication, Springer, Lecture Notes in Computer Science, LNCS-2091 Halmstad, Sweden, pp. 90-95, 2001

[18]Phillips P., Moon H., Rizvi S. A. and Rauss J., “The FERET evaluation methodology for face recognition algorithms”. IEEE Trans. Pattern Analysis Machine Intelligence, Gaithersburg, MD, USA, vol 22, pp.1090-1104, 2000. http://dx.doi.org/10.1109/34.879790

[19] D. L. Swets, J. Weng, “Using discriminant eigenfeatures for image retrieval”, IEEE Transactions on Pattern Analysis and Machine Intelligence. Aug 1996, Sioux Fall, SD, USA, Vol 18, pp 831- 836, 1996. http://dx.doi.org/10.1109/34.531802

\section{Copyright Disclaimer}

Copyright reserved by the author(s).

This article is an open-access article distributed under the terms and conditions of the Creative Commons Attribution license (http://creativecommons.org/licenses/by/3.0/). 